

\title{
Multi-Disciplinary Analysis for Future Launch Systems Using NASA's Advanced Engineering Environment (AEE)
}

\author{
D. Monell* \\ NASA Marshal Space Flight Center, Huntsville, AL 35812 \\ D. Mathias ${ }^{\dagger}$, and J. Reuther ${ }^{\ddagger}$ \\ NASA Ames Research Center, Moffett Field, CA 94035 \\ and \\ M. Garn \\ NASA Langley Research Center, Hampton, VA 23681-2199
}

\begin{abstract}
$\underline{\text { Abstract }}$
A new engineering environment constructed for the purposes of analyzing and designing Reusable Launch Vehicles (RLVs) is presented. The new environment has been developed to allow NASA to perform independent analysis and design of emerging RLV architectures and technologies. The new Advanced Engineering Environment (AEE) is both collaborative and distributed. It facilitates integration of the analyses by both vehicle performance disciplines and life-cycle disciplines. Current performance disciplines supported include: weights and sizing, aerodynamics, trajectories, propulsion, structural loads, and CAD-based geometries. Current life-cycle disciplines supported include: DDT\&E cost, production costs, operations costs, flight rates, safety and reliability, and system economics. Involving six NASA centers (ARC, LaRC, MSFC, KSC, GRC and JSC), AEE has been tailored to serve as a web-accessed agency-wide source for all of NASA's future launch vehicle systems engineering functions. Thus, it is configured to facilitate (a) data management, (b) automated tool/process integration and execution, and (c) data visualization and presentation. The core components of the integrated framework are a customized PTC Windchill product data management server, a set of RLV analysis and design tools integrated using Phoenix Integration's Model Center, and an XML-based data capture and transfer protocol. The AEE system has seen production use during the Initial Architecture and Technology Review for the NASA $2^{\text {nd }}$ Generation RLV program, and it continues to undergo development and enhancements in support of its current main customer, the NASA Next Generation Launch Technology (NGLT) program.
\end{abstract}

\section{Introduction}

The NASA Next Generation Launch Technology $(\text { NGLT })^{[1]}$ program encapsulates the agency's longterm space transportation strategic vision and technology development effort. This NASA program places importance on improving access to space for both governmental and commercial applications. It includes the efforts to mature the technologies and design the launch systems needed for the near term (10-15 year), mid-term (15-20 year) and far term (25 years and beyond) time frames. Its primary objective is to develop architecture and technology roadmaps for the future of launch systems at NASA.
The AEE system presented in this paper is currently focused on support of the NGLT program. The main effort is to fund the development of architectures and technologies for more reliable, safer launch capabilities at significantly lower cost than the Space Shuttle. Improving upon the current safety and cost metrics of the Space Shuttle is seen as an enabler for worldwide scientific exploration and commercial development of space. Previously, AEE was used in support of NASA's $2^{\text {nd }}$ Generation Reusable program. The $2^{\text {nd }}$ Gen. RLV program was a joint NASA/space industry effort, where industry performed the design and development functions, and NASA assessed the results.

\footnotetext{
Integrated System Engineering and Analysis Office Manager

${ }^{\dagger}$ AEE Advanced Tool Development Lead

${ }^{\ddagger}$ AEE Technical Lead, Associate Fellow AIAA

${ }^{\S}$ AEE Implementation Lead
} 
Under NASA's current NGLT program to develop future reusable launch systems, the Integrated Systems Engineering and Analysis (ISEA - VS30) office at Marshall Space Flight Center is tasked with providing overall systems analysis, design and engineering functions. The ISEA office is divided into four areas: the Systems Analysis (SA) project, the Systems Requirements and Analysis group, the Technologies Analysis and Assessment group, and the Advanced Engineering Environment (AEE) project. It is the job of SA to define and assess future launch architectures and evaluate emerging technologies that might be applicable to these architectures. The definitions of these architectures and the corresponding technology assessments are to be comprehensive, not only treating system performance capabilities but evaluating the complete system life-cycle parameters including costs, operations, support, maintainability, reliability and economics.

The Technology Analysis and Assessment group will determine the technologies that need to be assessed and the technology performance metrics that need to be tracked. The actual technology assessments as applied to NASA reference launch architectures will be performed by the Systems Analysis (SA) project. Development of the system requirements for near, mid and far term architectures as well as the metrics to track these requirements and their connection to the analysis of technologies and architectures will be the job of the Systems Requirements and Analysis group. Finally, it is the job of the AEE project to ensure that a software system is available to support the other three projects/groups and provide the ISEA office the ability to develop the roadmaps that are needed by NGLT. Figure 1 depicts the relationship of AEE to its elements, its inputs and outputs, and its customers.

An important role of the SA project is the accurate assessment of emerging architectures and technology developments. These assessments must be able to determine the validity and merits of all the funded architectures and technologies. The idea is for NASA to develop realistic roadmaps for both future launch architectures and the advanced technologies that will be necessary to enable them. Without such efforts, a development path that ensures safer and cheaper access to space cannot be established.

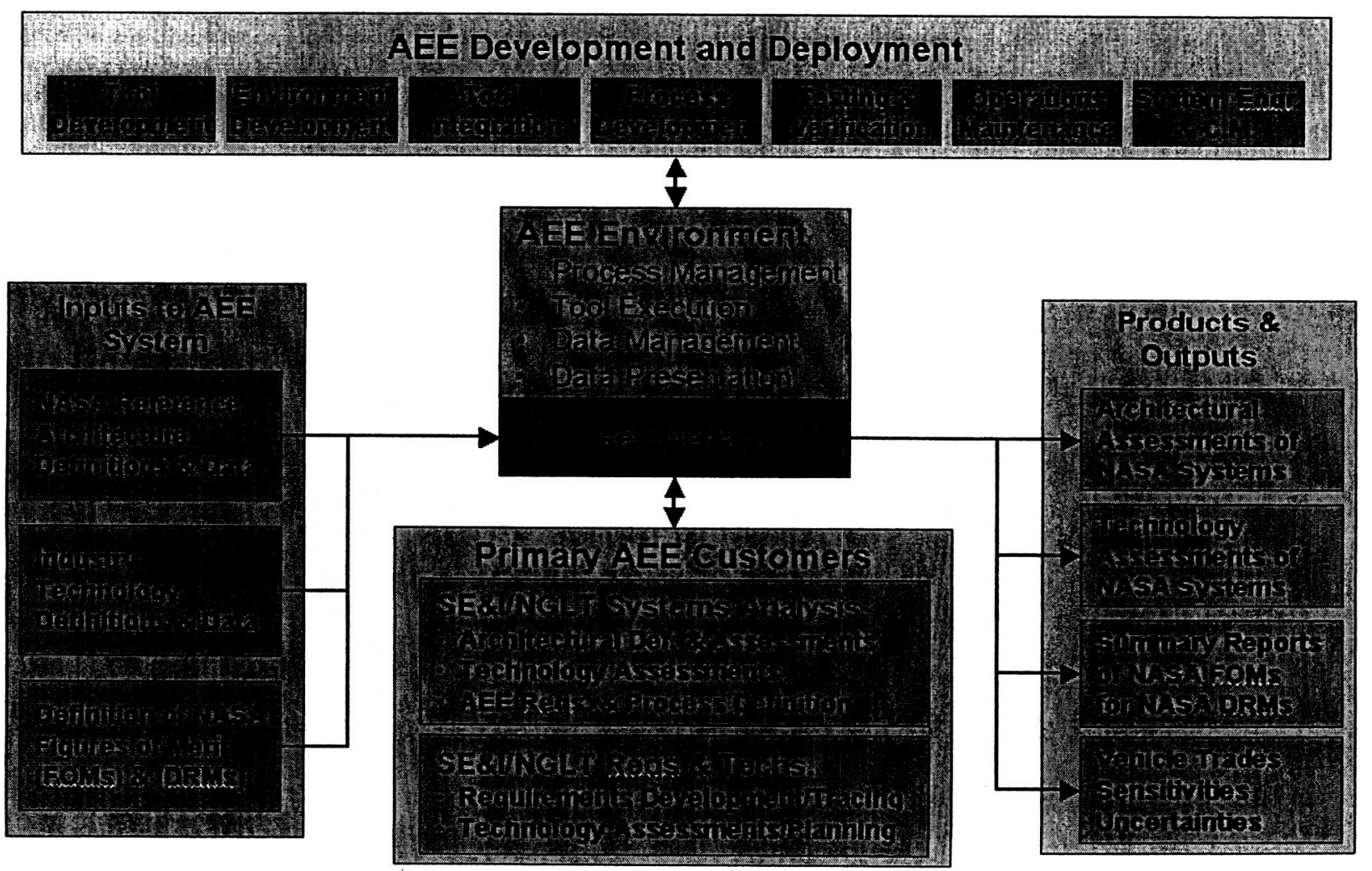

Figure 1: AEE relationship of organization, customers, inputs and outputs 